\title{
Resonant Gaps in the Scattered Cometary Population of the Trans-Neptunian Region
}

\author{
Tanya Taidakova \\ Crimean Astrophysical Observatory, Ukraine
}

\section{Leonid M. Ozernoy}

5C3, School of Computational Sciences and Department of Physics $\mathcal{G}$ Astronomy, George Mason U., Fairfax, VA 22030-4444, USA

Nick N. Gorkavyi

$N R C / N A S ; N A S A / G S F C$, Greenbelt, MD 20771, USA

\begin{abstract}
Our numerical simulations of the Edgeworth-Kuiper Belt objects gravitationally scattered by the four giant planets accounting for mean motion resonances reveal numerous resonant gaps in the distribution of the scattered population.
\end{abstract}

Available numerical simulations indicate that gravitational scattering of the EKBOs by the four giant planets might explain the transport of comets from the trans-neptunian region all the way inward, down to Jupiter, as well as the origin of the so called scattered disk (for a review, see Malhotra et al. 1999). Our recent simulations exploring both the spatial and phase structure of cometary populations beyond Jupiter (Ozernoy, Gorkavyi \& Taidakova 2000 $\equiv$ OGT) deal with 36 test bodies, initial positions of which were taken close to the known EKBOs so that their orbits intersected Neptune's orbit. Positions of all the scattered test bodies were traced for $\sim 0.5$ Gyrs and recorded each revolution of Neptune about the Sun, i.e. $165 \mathrm{yrs}$, totalling $\sim 2.8 \cdot 10^{7}$ positions. This describes in great detail the spatial area as well as the phase structure of the cometary population beyond Jupiter.

Our simulations reveal that (i) each giant planet dynamically controls a cometary population called the "cometary belt" (a well-known "cometary family', such as the Jupiter family comets, is just a visible part of the cometary belt); and (ii) comets avoid resonant orbits so that the belts contain numerous gaps in the $(a, e)$ - and $(a, i)$-spaces similar to the Kirkwood gaps in the main asteroidal belt. We have found that a numerous resonant gap structure is maintained in the scattered cometary population far beyond the Neptunian belt.

Figure 1 shows how the distribution of the scattered bodies in semimajor axis, $a$, evolves on a time scale of 0.5 Gyrs. Every bin of $\Delta a=0.1 \mathrm{AU}$ was visited by each of the test bodies, on average, $\sim 500$ times, which confirms a strong level of chaos in the scattered population. Our following findings are worth mentioning:

(i) Numerous gaps at the resonances with Neptune are well pronounced; (ii) A robust resonant structure of gaps is rapidly established; (iii) Unlike the distri- 

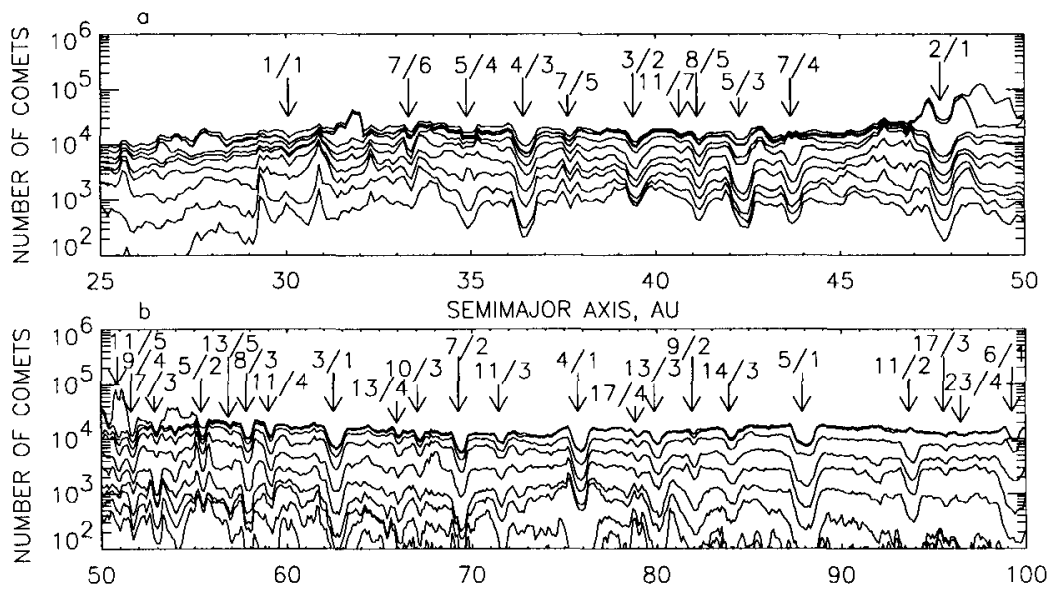

Figure 1. Evolution of the distribution of the simulated scattered comets in semimajor axis: $\mathbf{a}$ for comets with $25<a<50 \mathrm{AU}$ and $\mathbf{b}$ for comets with $50<a<100 \mathrm{AU}$. The number of comets is given between $a$ and $a+\Delta a$, where $\Delta a=0.1 \mathrm{AU}$. The arrows indicate the various resonances with Neptune. Ten distributions, from below to the top, are shown at $1,2,4,8,16,32,64,128,256$, and $500 \mathrm{Myrs}$, respectively.

bution of the scattered objects in heliocentric distance, which has a maximum near the Neptunian orbit (Malhotra et al. 1999, OGT), the distribution in semimajor axis appears rather uniform; (iv) There are appreciable accumulations of the scattered comets near the resonances well outside the zone of a strong gravitational scattering (e.g., near the 2:1 resonance). Those accumulations (called 'diffusive' ones in OGT) are produced by slowing down the diffusion as the comet's distance of pericenter turns out to be outside the planetary orbit.

\section{References}

Malhotra, R., Duncan M., \& Levison, H. 1999, astro-ph/9901155

Ozernoy, L.M., Gorkavyi, N.N., Taidakova, T., 2000, Planetary Space Sci. 48, 993 